\title{
EL TRABAJO SOCIAL COMO AGENTE DE IGUALDAD: HISTORIA E INTERVENCIÓN SOCIAL DESDE LA PERSPECTIVA DE GÉNERO ${ }^{1}$
}

\author{
Francisco Antonio Castaño Rodríguez ${ }^{2}$
}

\begin{abstract}
El Trabajo Social como agente de igualdad: Historia e intervención social desde la perspectiva de género

Resumen: El Trabajo Social ha sido y es una profesión de mayoría femenina, tanto en su ejercicio como en la recepción de servicios y recursos. La intervención social se convierte así en un escenario en el que desarrollar la perspectiva de género al redefinir la realidad y dar a conocer las desigualdades existentes en la sociedad. Si bien es cierto que la perspectiva de género en la teoría y práctica del Trabajo Social no son comunes, esta profesión, ejercida desde una óptica feminista, establece estrategias transversales que producen un giro epistemológico y un cambio en el paradigma de la acción social.

Palabras clave: Estudios de género, Trabajo Social, Intervención Social, Historia del Trabajo Social.
\end{abstract}

Social Work as Agent of Equality: The History of Social Intervention from a Gender Perspective

Abstract: Social Work has traditionally been a female profession both in its performance and in the perception of services and resources. Social intervention thus becomes a setting where a gender perspective can be applied to redefine reality and disclose existing social inequalities. Applying a gender perspective to Social Work has not been a common practice, yet, this profession, if undertaken with a feminist agenda, allows for the establishment of cross-sectional strategies that produce an epistemological shift.

Keywords: Gender Studies, Social Work, Social Intervention, Social Work History.

\section{Introducción.}

Desde sus inicios, a través de obras caritativas y benéficas, hasta su institucionalización como estudios universitarios, el Trabajo Social ha evolucionado y se ha tecnificado con una relevante participación de la mujer. El fin esencial de esta profesión se enmarca en los principios recogidos en la Declaración Universal de los Derechos Humanos y está encaminado a promover el cambio social, a la resolución de problemas y necesidades sociales y, en última instancia, a conseguir el bienestar de los

\footnotetext{
${ }^{1}$ Fecha de recepción: 01/12/2016.

Fecha de aceptación: 22/12/2016.

2 Trabajador Social. Máster en Estudios de Género, Mujer Cultura y Sociedad, Universidad de Almería; $₫$ francasrod@hotmail.com.
} 
seres humanos. Por este motivo, feminismo y Trabajo Social no pueden existir desligados el uno del otro, ya que ambos abogan por la igualdad y la justicia social. Sin embargo, el primero continúa, a día de hoy, luchando por hacerse un hueco en los espacios de intervención social.

Como se expondrá a lo largo del presente documento, el sistema patriarcal, imbricado en todas las profesiones e instituciones, se encuentra presente también en el ejercicio y desarrollo del Trabajo Social. Este hecho ocasiona que, a pesar de su situación privilegiada para denunciar las situaciones de desigualdad, esta profesión no cuestione los motivos por los que las mujeres son las principales usuarias de este tipo de servicios y recursos, que no analice el impacto de los proyectos de intervención atendiendo a indicadores de género, e incluso, que dentro del ejercicio de la profesión se produzcan situaciones que pueden ser explicadas dentro del marco de las teorías de la división sexual del trabajo.

\section{La relación del Trabajo Social y del Feminismo en la literatura científica.}

En los años 80 del siglo pasado, comienzan a publicarse los primeros estudios que abordan la relación de feminismo y Trabajo Social. El más destacado de ellos es Trabajo Social Feminista, de Lena Dominelli y Eileen Macleod, publicado por primera vez en 1989. Ambas autoras analizan la presencia feminista en la definición de los problemas y las necesidades sociales, así como la contribución que el Trabajo Social aporta al Feminismo en general. Sin embargo, a este estudio le preceden Defining Feminist Social Work (Collins 1986), Not for women only: Social work practice for a feminist future (Bricker-Jenkins y Hooyman 1986) y Feminist visions for social work (Van Den Bergh y Cooper 1986). Los referidos trabajos coinciden en destacar que los valores y la ética del Trabajo Social están muy cercanos a los principios feministas, así como en la necesaria incorporación de la perspectiva de género en la intervención social (Alcázar-Campos 2014: 27). 
Viscarret (2007: 208-213) enmarca el Trabajo Social Feminista dentro del modelo de intervención crítico-radical, que sostiene que no son las circunstancias personales y sociales a las que se enfrentan las personas las responsables de su situación de desigualdad social, sino que esta realidad estaría determinada por la propia opresión de las estructuras sociales. En este sentido, el Trabajo Social Feminista quedaría definido como "una forma de Trabajo Social práctico que tiene la desigualdad de género y la eliminación de la misma como punto de partida para trabajar con mujeres, buscando promover el bienestar de la mujer tal y como lo definan las propias mujeres" (Dominelli 1997, citado en Viscarret 2007: 215).

La definición anterior quedaría en cierto modo limitada, ya que establece el Trabajo Social Feminista como una forma exclusivamente "práctica"; es decir, una forma de intervención sin un marco teórico propio. A este respecto, Fernández-Montaño (2015: 24) sostiene que la intervención social (la "práctica" del Trabajo Social) se encuentra insertada en unas instituciones y en unos valores que colaboran en el sostenimiento del sistema patriarcal, motivo por el cual es necesario que el ejercicio de la profesión tenga también carácter investigador. De este modo, se conseguiría cuestionar las estructuras sociales y visibilizar y analizar las manifestaciones sexistas que ahondan en el origen de los problemas y necesidades sociales. En esta misma línea, Orme (2002: 806) sostiene que el feminismo posee la capacidad de denunciar situaciones de discriminación y debatir significados y conceptos desde un enfoque transformador. Igualmente, Azpeitia Armán (2003: 162) reclama el uso de teorías feministas para la construcción de teorías en Trabajo Social, siendo el punto de partida fundamental para iniciar el "proceso de recuperación de la mujer como objeto y sujeto de investigación".

Otro tema de análisis lo constituye el debate en torno a la identidad profesional de las trabajadoras sociales y la división sexual del trabajo. Bañez Tello (1997: 159-162) sostiene que el Trabajo Social nace para posibilitar el control social de la pobreza y la mujer por medio de la propia función legitimadora de la profesión: la atención, los cuidados, la alfabetización, la socialización de menores y, en definitiva, la reproducción social, son funciones que tradicionalmente ha asumido la mujer en el marco de la esfera doméstica, por lo que la profesión sería una prolongación de sus cualidades femeninas. 
En la actualidad, a través de la construcción y el mantenimiento de los estereotipos de género que vinculan las emociones y los cuidados con la imagen de la mujer, el Trabajo Social, al igual que otras profesiones como la enseñanza o la enfermería, contarían con una mayor presencia femenina. Este es el motivo por el que Berasaluze Correa (2009: 136) afirma que el Trabajo Social es considerado una semiprofesión, porque "al ser una actividad que se ocupa de ayudar a las personas y es ejercida fundamentalmente por mujeres, se ha percibido como una prolongación del rol femenino".

Para finalizar este epígrafe, resulta necesario mencionar los sectores y ámbitos de intervención relacionados con las necesidades y problemas específicos de la mujer que han captado mayor interés por parte de quienes se dedican a la investigación y docencia del Trabajo Social: violencia de género y desigualdad en el empleo en primer y segundo término respectivamente, seguido por mujeres inmigrantes $y / o$ en contextos de prostitución. De forma más reciente, y debido a la persistente situación de crisis económica, la progresiva feminización de la pobreza es otro de las cuestiones objeto de estudio y análisis.

\section{Las mujeres en la construcción del método en Trabajo Social y el papel del Feminismo.}

Los estudios de género hacen hincapié en la influencia silenciada de las mujeres en los distintos ámbitos del saber. La construcción del Trabajo Social, como profesión y disciplina, ha estado constituida por diferentes fases, en las cuales siempre hubo mujeres dispuestas a intervenir ante los problemas sociales, estudiar las vías adecuadas para resolverlos e institucionalizar estas enseñanzas de cara a mejorar la intervención social con sus distintas aportaciones. En relación a lo anterior, se expondrán las referidas fases de construcción del Trabajo Social propuestas por Barrera Algarín (2005: 46) ${ }^{3}$ y se completarán con las aportaciones de sus precursoras y del feminismo.

\footnotetext{
${ }^{3}$ El autor toma como base las fases establecidas por Boris (1983) y García Alba y Melián Melián (1993).
} 
3.1. Etapa pre-técnica. Fase benéfica y caridad pública.

Tiene su inicio en la Edad Media y se prolongó hasta la Revolución Industrial. La Iglesia fue la principal garante de asistencia, pues se consideró la atención a pobres e indigentes como un vehículo para acercarse a Dios. Destaca la congregación religiosa de las Hijas de la Caridad de San Vicente Paul, cofundada por Santa Luisa de Marillac (1591-1660), quien reformó la atención a las personas necesitadas en las instituciones religiosas de Francia, e instruyó, asimismo, a otras mujeres para una acción rigurosa en el servicio a las personas en situación de vulnerabilidad.

\subsection{Etapa técnica. Fase de Asistencia Social.}

Con el surgimiento y afianzamiento de la sociedad industrial, determinados grupos sociales (especialmente procedentes de la burguesía urbana) ponen en marcha la denominada "Cuestión Social". Consideran que las antiguas formas de caridad y filantropía resultan insuficientes ante la extensión de las situaciones de pobreza que conlleva la Revolución Industrial. Demandan mayor intervención estatal y políticas públicas en materia de higiene, salud, alfabetización, vivienda y mejora de las condiciones de vida de la clase obrera. Se busca, en definitiva, el bienestar y la mejora de los colectivos oprimidos de una incipiente economía capitalista.

Como organizaciones, resultan relevantes la Charity Organization Society ${ }^{4}$, en Estados Unidos, y el Settlement Movement ${ }^{5}$, en Reino Unido. Ambas responden al deseo de una mejor administración de los recursos disponibles y a la importancia de la relación interpersonal en los procesos de ayuda, entendidos como un proceso en el que la persona que recibe la atención y los recursos es la precursora de su propio cambio.

La primera de ellas fue fundada por Josephine Shaw Lowell (1843-1905), quien creó un registro central de personas que solicitaban o recibían asistencia y elaboró un plan de intervención basado en una completa investigación de los problemas sociales (Barrera

\footnotetext{
${ }^{4}$ La Charity Organization Society suele ser nombrada en la literatura científica por las siglas COS.

${ }^{5}$ En castellano, "Movimiento de los Establecimientos".
} 
Algarín, 2005: 43). Esta organización contó entre sus colaboradoras con dos mujeres que resultarán relevantes para el desarrollo metodológico y científico del Trabajo Social y, al mismo tiempo, serían las artífices de la creación de escuelas para la formación de

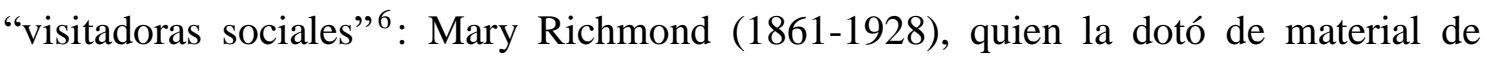
enseñanza al ofrecer conferencias y seminarios sobre objetivos y métodos para una rigurosa intervención, y Octavia Hill (1838-1912), cuyo trabajo se centró especialmente en las insalubres condiciones de las viviendas de los barrios de clase obrera a través del "sistema Hill de Viviendas"7. Además, estableció una serie de normas para el registro de los diferentes casos en lo que es la forma primitiva de los actuales instrumentos básicos de la profesión: la ficha social y el informe social (Barrera Algarín 2005:42-45).

La segunda organización citada fue la fundada por el matrimonio formado por Samuel Barnett (1844-1913) y Henrietta Barnett (1851-1936). En la misma línea que Josephine Shaw Lowell, abogaron por la investigación de las causas subyacentes a la situación de necesidad social como vía fundamental para asistir a quienes demandaban ayuda, así como en la estricta confidencialidad de los procesos de intervención. A través de sus casas de rehabilitación y con la presencia de personas de la burguesía londinense, ofrecían servicios de alfabetización y desarrollo cultural mediante el contacto directo entre personas de diferentes clases sociales. Es importante resaltar este hecho, pues creían que a través de estas acciones la clase adinerada y dirigente obtenía el conocimiento necesario de las condiciones sanitarias, higiénicas y educativas de la creciente clase obrera de la sociedad industrial que ayudaría a emprender una reforma social.

En España, por su parte, destaca la figura de Concepción Arenal (1820-1893), quien publicó en 1861 La beneficencia, la filantropía y la caridad y en 1863 El visitador del pobre, dos obras de especial relevancia para el Trabajo Social. En la primera de ellas mostró "su idea de compaginar eficazmente la beneficencia pública y la caridad privada,

\footnotetext{
${ }^{6}$ Estas mujeres eran el nexo entre las instituciones de asistencia social y las clases proletarias. Su labor principal era detectar las necesidades y problemas sociales de la familia en cuestión y buscar los recursos adecuados para lograr mejorar sus condiciones de vida.

${ }^{7} \mathrm{El}$ "sistema "Hill de Viviendas" consistía en reformar edificios antiguos y en ruinas en pisos destinados a las familias que trabajaban en las fábricas, realizando siempre un estudio previo y una posterior selección de aquellos casos que, a pesar de su situación de vulnerabilidad social, mostrasen actitudes y aptitudes para el cambio (Barrera Algarín 2005: 42).
} 
dentro de una acción social organizada con distribución de competencias" (Capilla Pérez 2001: 156) para una correcta administración de los recursos; en la segunda, escrita con la finalidad de formar a las visitadoras de las Hijas de la Caridad de San Vicente de Paul, expuso que para alcanzar la autonomía personal y social cada persona debe tomar conciencia de sí misma. La función de la visitadora sería ayudar a desarrollar integralmente las facultades morales e intelectuales de quien recibe la acción asistencial (Eito Mateo 2012: 251-252).

Esta etapa se caracterizó por su riqueza, pues gracias a las contribuciones de las mujeres anteriormente mencionadas, se fueron creando los marcos metodológico y ético que la disciplina irá perfeccionando posteriormente, así como los instrumentos de trabajo primitivos del Trabajo Social. Asistimos, además, al desarrollo de los métodos actuales de la profesión: Trabajo Social con Casos, Trabajo Social con Grupos y Trabajo Social Comunitario.

\subsection{Etapa Pre-científica. Fase de Servicio Social.}

Auspiciada por las novedades metodológicas y técnicas introducidas en la fase anterior, esta etapa se extiende desde los últimos años del siglo XIX hasta la mitad del siglo XX. Surge el Trabajo Social como ciencia y disciplina académica de la mano de Mary Richmond, se avanza en su propio modelo de acción práctica y se dan los principales debates en torno el método científico de la profesión.

Mary Ellen Richmond es la autora clave en la configuración de esta nueva profesión (Miranda Aranda 2011: 35). En 1917 publicó su libro Diagnóstico Social, donde a través de una rigurosa investigación y a partir de la sistematización de experiencias profesionales, expuso los principios básicos de la acción profesional, los criterios y procedimientos utilizados para su análisis y los principales conceptos teóricos que la sustentan (Travi 2011: 73-75). En el referido trabajo explicó, además, el proceso que se debe seguir a la hora de tener en cuenta las necesidades y problemas sociales desde una perspectiva multidimensional, lo que conlleva realizar tanto una acción directa a 
través de la comprensión de la individualidad del ser humano, como una acción indirecta, por medio de los recursos, riesgos e influencias del entorno social (Barriga Muñoz y Martínez Alonso 2011: 121). En 1922 publicó Qué es el Trabajo Social de Casos, donde expuso cómo el ser humano adquiere la personalidad a través de sus relaciones con la sociedad, por lo que la función del Trabajo Social sería descubrir las relaciones sociales que más le convenían y recurrir a los distintos agentes para procurárselas. Estableció la creación de un programa social que igualase las posibilidades de todas las personas en una acción conjunta profesional-persona beneficiaria (Miranda Aranda 2011: 43). Mary Richmond concebía a la persona beneficiaria como un elemento activo en el camino que procura su transformación, junto a la necesaria intervención de otros agentes de su entorno comunitario que garantizasen su inserción social.

En resumen, la metodología de trabajo y las bases científicas de Mary Richmond abrieron una nueva vía de comprensión de los factores que subyacen a las necesidades y problemas sociales, y donde la búsqueda del desarrollo de la personalidad y de la capacidad de resolver los problemas por sí mismo o misma serían el auténtico proyecto de intervención. Por todo lo expuesto anteriormente, es por lo que Mary Richmond recibió el título de Doctora "Honoris Causa" por el Smith College al "sentar las bases científicas de una nueva profesión” (Barriga Muñoz y Martínez Alonso 2011: 113-118).

Jane Addams (1860-1935), interesada en la línea de trabajo del "Movimiento de los Establecimientos", fundó las Hull House, convirtiéndose en uno de los primeros centros estadounidenses en atender a personas sin recursos que proporcionaron herramientas para su inserción social a través de una atención integral de la persona. Ligada también al movimiento sufragista, presidió la Liga Internacional de Mujeres Pro Paz y Libertad y recibió, en 1931, el Premio Nobel de la Paz (Fombuena Valero 2006: 138-139).

Finalmente, Alice Salomon (1872-1948) se constituyó en una de las pioneras en la docencia de la disciplina gracias a la combinación de la Pedagogía y del Trabajo Social, impulsó la creación de encuentros internacionales y participó en el desarrollo de un enfoque crítico y científico del bienestar social (Barrera Algarín, 2005: 44). 


\subsection{Etapa científica. Fase de Trabajo Social.}

Comienza a partir de la mitad de la centuria pasada y se caracteriza por la indagación de las relaciones causales que subyacen a las necesidades sociales. En esta última etapa reconocida, los países occidentales han implantado, desde diferentes enfoques e ideologías, una serie de políticas públicas que sostienen un Estado de Bienestar. El Trabajo Social comienza a quedar relegado a la mera gestión de recursos, ayudas públicas y otras tareas de índole burocrática, olvidando la investigación y la transformación de la realidad social (Barrera Algarín 2005: 49). Se produce, entonces, una revisión y reconceptualización de la disciplina por no ajustarse a la realidad por la que la profesión nace.

Este proceso de reajuste de la profesión coincide con el auge del movimiento Feminista de la Segunda Ola, que pone de relieve la importancia y similitudes de ambos y sostiene que el Trabajo Social es un medio adecuado para el desarrollo del Feminismo y viceversa. La intervención social, desde un enfoque feminista, identifica como objetivo final la eliminación de la opresión de la mujeres, la redefinición de los roles de género y el desarrollo del potencial de cada mujer. Para ello, afirman las trabajadoras sociales feministas, es necesario recuperar la esencia de los inicios de la profesión y la metodología empleada por las pioneras. Así, ejercer el Trabajo Social Feminista supone (Viscarrent 2007: 214-218):

- Clarificar las necesidades y problemas diferenciados de hombres y mujeres.

- Luchar por la igualdad de hombres y mujeres y contra la opresión de éstas.

- Dar voz y escuchar a las mujeres para buscar soluciones colectivas que respeten su individualidad.

- Utilizar sus contribuciones y experiencias para dar sentido a la realidad social en la que están insertas.

- Ganar en autoconocimiento al explorar cómo cada una de las mujeres ha usado sus conocimientos y experiencias ante determinadas situaciones.

- Estudiar distintas alternativas de intervención y formular planes de acción consensuados. 
- Crear redes comunitarias que desarrollen las capacidades y potencial de las mujeres.

\section{El Trabajo Social desde la perspectiva de género en la actualidad.}

Los supuestos de Viscarrent mencionados en el epígrafe anterior suponen un marco ideal de trabajo más que una realidad, ya que no son, en la mayoría de los casos, tenidos en cuenta a la hora de elaborar un proceso de intervención social. Entre las razones para justificar este hecho encontramos, en primer término, la ausencia de formación en materia de género entre quienes ejercen esta actividad profesional. La introducción de una asignatura, no siempre de carácter obligatorio, sobre feminismo, género y mujer en el currículo académico de Trabajo Social se produjo con la sustitución de las diplomaturas por los nuevos estudios de grado. Esto explica, a su vez, la escasa existencia de investigaciones llevadas a cabo desde el ámbito universitario. En segundo lugar, la elevada ratio profesional/habitante ${ }^{8}$, que a menudo obliga a las trabajadoras y trabajadores sociales a establecer actuaciones genéricas ante una misma situación de necesidad social. Este hecho supone, por tanto, obviar la perspectiva de género durante el proceso de trabajo social. Mención aparte tienen las acciones formativas destinadas a mujeres llevadas a cabo desde las distintas Administraciones Públicas locales a través de sus áreas de cultura y servicios sociales. Por lo general, estos talleres se caracterizan por ser breves, de escasa continuidad en el tiempo y limitados a la disponibilidad presupuestaria, lo que impide la consecución de unos objetivos a largo plazo.

Como se ha expuesto a lo largo del presente documento, el Trabajo Social como profesión y como disciplina dentro de las Ciencias Sociales, presenta una particularidad respecto al resto de profesiones y disciplinas: tiene origen femenino y continúa siendo ejercido, en mayor medida, por mujeres (Azpeitia Armán 2003: 151). En esta misma línea, Berasaluze Correa y Ovejas Lara (2009: 10) afirman que el 95\% de quienes ejercen el Trabajo Social en nuestro país son mujeres.

\footnotetext{
${ }^{8}$ Según Celdrán Martínez (2015: 145), dependiendo de la Comunidad Autónoma, encontramos una ratio que comprende desde los 3.000 habitantes por profesional hasta los 7.500.
} 
La introducción de las teorías feministas, a través de sus diferentes corrientes, aportaron una nueva forma de analizar la realidad y pusieron de manifiesto la categoría “mujer" como sujeto y objeto de análisis. Sin embargo, en España, la incorporación de la perspectiva de género al Trabajo Social ha sido tardía si se compara con otros países y otras profesiones y disciplinas. Además, el hecho de tener una mayoría de mujeres ejerciendo y otra mayoría recibiendo servicios y recursos no significa que se trabaje desde la perspectiva de género, pues "nuestra disciplina y profesión no ha hecho el esfuerzo suficiente para incorporar la perspectiva de género en su desarrollo" (Berasaluze Correa y Ovejas Lara 2009: 10). Cabe preguntarse, pues, el motivo. Por ello se exponen en líneas sucesivas las posibles respuestas aportadas por la literatura científica.

Durante el proceso de profesionalización, tecnificación e integración en el mundo laboral y universitario, tiene lugar el establecimiento de un sistema público de protección y bienestar social, algo que autoras como Azpeitia Armán (2003: 164) consideran que contribuyó a silenciar a esa mayoría femenina e hizo olvidar la cuestión de género. Ahondando aún más, Báñez Tello (1997: 185) critica las teorías y modelos de intervención que no profundizan en el hecho de que sean las mujeres las mayores solicitantes de este tipo de servicios, ya que en ocasiones sus demandas no están relacionadas consigo mismas, sino con algún miembro de la familia o con el comportamiento disfuncional de ésta en su conjunto. Es decir, la profesión no cuestiona las relaciones de género en las relaciones familiares, ni incide de forma estructural en los procesos de feminización de la pobreza. En esta misma línea argumental, otra posible explicación al escaso eco de las teorías feministas y de género en el Trabajo Social se debe al androcentrismo existente en los centros de enseñanza universitarios. Según Guzmán Stein (33-34 y 36) el sexismo está muy arraigado en la enseñanza de la profesión, ya que el currículo no está estructurado de tal forma que incorpore modelos de intervención alternativos que pongan de manifiesto los intereses de las mujeres y que lleven a una comprensión y a un análisis de los problemas sociales con perspectiva de género.

Otras autoras, como Nebreda Roca (2009: 68), afirman que este hecho va unido también a la propia incorporación de los hombres a la profesión, que tuvo lugar 
reproduciendo los estereotipos de género. Así, mientras las mujeres se encargaban del trabajo directo con las personas beneficiarias (los cuidados), los hombres gestionaban y administraban las instituciones, tanto públicas como privadas, de asistencia social. En este sentido, las teorías de la división sexual del trabajo, por la que los hombres se ocupan de la producción y las mujeres de la reproducción, considerarían al Trabajo Social una extensión de las cualidades femeninas (Azpeitia Armán 2003: 150). En esta línea, Roldán García, Leyra Fatou y Contreras Martínez (2012: 43) afirman que el mercado laboral del Trabajo Social se caracteriza por una segregación laboral en la que los varones ocupan los cargos de representación y toma de decisiones y las mujeres se topan, una vez más, con el "techo de cristal" que les impide optar a los puestos directivos, a pesar de suponer una mayoría en el ejercicio de la profesión.

\section{Conclusiones}

Como puede observarse, los postulados de las teorías feministas aplicadas al Trabajo Social no difieren de lo propuesto por las pioneras de la profesión: trabajar de forma conjunta con la persona objeto de intervención, escuchar lo que tiene que decir al respecto de su situación, tener en cuenta sus experiencias y entorno social y, finalmente, consensuar unos objetivos y un plan de actuación, pues únicamente si la persona beneficiaria se reconoce a sí misma durante el proceso de cambio y transformación, el resultado será exitoso. El Trabajo Social, al igual que el Feminismo, hace hincapié en la diversidad y en las distintas formas de ser mujer, ya que una misma realidad social, cultural e histórica es diferente para una mujer u otra.

En los países occidentales, con el desarrollo del Estado de Bienestar, se ha ido arraigando una concepción de esta profesión burocrática y eminentemente gestora de recursos y ayudas públicas. Esto se debe a la identificación de los sistemas públicos de Servicios Sociales como única expresión de la profesión. Actualmente, y ante una situación de crisis financiera que ha mermado los recursos presupuestarios destinados a las áreas de igualdad y bienestar social de las distintas Administraciones Públicas, son las ONG's quienes están llevando a cabo la labor de denuncia social y dinamización del 
tejido comunitario. Este puede ser el escenario perfecto para reestructurar los modelos de intervención, realizar una relectura de las pioneras de la profesión y de las aportaciones de las teorías feministas de los años 70 y 80 del siglo pasado.

Para ello es necesario, en primer lugar, incorporar los postulados del Feminismo al currículo universitario para una mejor comprensión de los problemas y necesidades sociales. Del mismo modo, resulta importante promover la dimensión investigadora de la profesión con líneas específicas de género que incidan en la situación de la mujer en los diferentes ámbitos de intervención social.

Para que el Trabajo Social sea de una forma efectiva y real un escenario de igualdad, las trabajadoras y trabajadores sociales deben aplicar una intervención desde la perspectiva de género: a la hora de realizar los diagnósticos, teniendo en cuenta las necesidades diferenciadas de las personas que componen el núcleo familiar y las del grupo en su conjunto; durante el proceso de planificación de objetivos y actividades, escuchando sus experiencias e intereses; y finalmente, estableciendo indicadores de evaluación diferenciados en clave de género como única vía para conocer el impacto real que las acciones profesionales tienen sobre la mujer.

Para finalizar, es necesario destacar a Bacete (2009: 100) cuando afirma que introducir la perspectiva de género en nuestra profesión aporta nuevos elementos de análisis de los problemas sociales y novedosas herramientas de intervención, debido a que una parte muy significativa de los problemas sociales tiene un evidente sesgo de género.

\section{Referencias bibliográficas}

Alcázar-Campos, Ana. 2014. "Miradas feministas y/o de género al Trabajo Social, un análisis crítico". Portularia, vol. 14(1): 27-34.

Azpeitia Armán, María Concepción. 2003. "Género e identidad profesional en los trabajadores sociales". Cuadernos de Trabajo Social, vol. 16: 147-170. 
Bacete, Ritxar. 2009. "La perspectiva de género de los hombres aplicada a la intervención social: el sexismo y la masculinidad hegemónica como problemas sociales". II Jornadas de Trabajo Social. Hacia una Intervención con Perspectiva de Género. Eds. Aranguren Vigo, Edurne y Villaño Murga, Gotzon. Vitoria: Universidad del País Vasco. 99-103.

Bañez Tello, Tomasa. 1997. "Género y Trabajo Social”. Acciones e investigaciones sociales 6: 151-188.

Barrera Algarín, Evaristo. 2005. Metodología del Trabajo Social. Sevilla: Aconcagua Libros.

Barriga Muñoz, Lourdes y Martínez Alonso, María Ángeles. 2011. "Mary Richmond en la perspectiva del Trabajo Social en España". Cuadernos de Trabajo Social, vol. 24: 113-121.

Berasaluze Correa, Ainhoa. 2009. "El devenir del Trabajo Social en clave de género". Zerbitzuan: Revista de Servicios Sociales, vol. 46: 133-140.

Berasaluze Correa, Ainhoa, y Ovejas Lara, Charo. 2009. "Prólogo". II Jornadas de Trabajo Social. Hacia una Intervención con Perspectiva de Género. Eds. Aranguren Vigo, Edurne y Villaño Murga, Gotzon, Vitoria: Universidad del País Vasco. 10-11.

Lima, Boris. 1983. Epistemología del trabajo social. Buenos Aires: Humanitas.

Capilla Pérez, Andrea. 2001. “Concepción Arenal: un enfoque desde el Trabajo Social”. Portularia 1: 155-170.

Celdrán Martínez, Diego. 2015. "La ratio por Trabajador Social en las Unidades de Trabajo Social”. Documentos de Trabajo Social, vol. 55: 144-160.

Dominelli, Lena y Eileen Macleod. 1999. Trabajo Social Feminista. Madrid: Cátedra.

Eito Mateo, Antonio. 2012. "La participación del usuario en el Trabajo Social. Una mirada desde el presente hacia el humanismo de Concepción Arenal". Acciones e Investigaciones Sociales, vol. 32: 245-255.

Fernández-Montaño, Patricia. 2015. "Trabajo Social Feminista: una revisión teórica para la redefinición práctica”. Trabajo Social Global. Revista de Investigaciones en Intervención Social, vol. 5(9): 24-39.

Fombuena Valero, Josefa. 2006. "La influencia de la dimensión género en el Trabajo Social". Cuadernos de Trabajo Social, vol. 19: 133-154.

García Alba, Jesús y Melián Melián, Jesús R. 1993. Hacia un nuevo enfoque del Trabajo Social. Madrid. Narcea.

Guzmán Stein, Laura. 2000. "Identidad profesional y sexismo en la formación en Trabajo Social”. Revista Costarricense de Trabajo Social, vol. 11: 31-39 
Miranda Aranda, Miguel. 2011. "Contexto de la actividad y el pensamiento de Mary Richmond”. Cuadernos de Trabajo Social, vol. 24: 35-45.

Nebreda Roca, María Isabel. 2009. "El Trabajo Social y las relaciones de género”. II Jornadas de Trabajo Social. Hacia una Intervención con Perspectiva de Género. Eds. Aranguren Vigo, Edurne y Villaño Murga, Gotzon, Vitoria: Universidad del País Vasco. 65-71.

Orme, John. 2002. "Social Work: Gender, Care and Justice". British Journal of Social Work 32: 799-814.

Roldán García, Elena, Begoña Leyra Fatou y Leticia Contreras Martínez. 2012. "segregación laboral y techo de cristal en Trabajo Social: análisis del caso español". Protularia, vol. 12(2): 43-56.

Travi, Bibiana. 2011. "Conceptos e ideas clave en la obra de Mary Ellen Richmond y la vigencia actual de su pensamiento". Política Social y Trabajo Social: desarrollo histórico y debates actuales. Ed. Miguel Miranda Aranda. Zaragoza: Universidad de Zaragoza. 69-87.

Viscarret, Juan Jesús. 2007. Modelos y métodos de intervención en Trabajo Social. Madrid: Alianza. 